\title{
Piezoelectric effects in the optical properties of strained InGaN quantum wells
}

\author{
L.-H. Peng ${ }^{\text {a) }}$ \\ Department of Electrical Engineering and Institute of Electro-Optical Engineering, National Taiwan \\ University, Taipei, Taiwan, Republic of China \\ C.-W. Chuang \\ Institute of Electro-Optical Engineering, National Taiwan University, Taipei, Taiwan, Republic of China \\ L.-H. Lou \\ Nichia-Unilux Corporation, Hsinchu, Taiwan, Republic of China
}

(Received 7 July 1998; accepted for publication 2 December 1998)

\begin{abstract}
We investigated the carrier-injection effects in the emission spectrum of strained GaN/InGaN/ $\mathrm{AlGaN}$ quantum well $(\mathrm{QW})$ blue emitters using a pulsed current excitation technique. Spectral blueshift as large as $80 \mathrm{meV}$ in the emission peak energy was observed as the injection current increases from $1 \mathrm{~mA}$ to $1 \mathrm{~A}$. Based on a self-consistent calculation that couples the Poisson equation with a wurtzite-type Rashba-Sheka-Pikus Hamiltonian, four important interactions are evaluated in order to determine the optical properties of InGaN QW. It is shown that the spectral redshifting caused by a piezoelectricity induced quantum confined Stark effect and carrier-induced band gap renormalization is counteracted by a blueshift due to the band filling and charge screening effects. The increase of InGaN QW emission peak energy and intensity with injected carriers suggests a dominant contribution from the latter in a band-to-band recombination process. (C) 1999 American Institute of Physics. [S0003-6951(99)01906-3]
\end{abstract}

Recent progress in the epitaxial growth, doping control, and device processing of indium gallium nitride $(\mathrm{InGaN})$ has made this material system a promising light emitter operated in the wavelength regime ranging from ultraviolet to visible. ${ }^{1}$ Success of Nichia's commercial high brightness blue/green light emitting diodes represents one such exciting achievement ${ }^{2}$ while the more recent reports of long lifetime continuous wave room temperature operation of InGaN laser diodes on sapphire ${ }^{3}$ and gallium nitride $(\mathrm{GaN})^{4}$ substrates have stimulated further scientific investigation. Despite the fact that InGaN is one of the most applicable materials used for short wavelength emitters, many of its novel optical properties such as carrier-induced spectral shifting ${ }^{5}$ still remain a great challenge to be resolved. Conventional wisdom has pursued a model analysis based on a wurtzite-type Rashba-Sheka-Pikus (RSP) Hamiltonian in calculating the band structures of InGaN-based quantum wells (QWs). ${ }^{6}$ In this method, intrinsic properties such as piezoelectricity and many-body effects are often neglected in the so-called free carrier model in order to grant a guideline on the device designing issues. Although simplistic in nature, considerable discrepancy has been found between the optical measurements and the calculated spectra. For example, an emission peak energy difference on the order of $200 \mathrm{meV}$ has been noted in a InGaN QW emitter when compared with a flatband model calculation. ${ }^{2}$

From the device physics point of view, it is desirable to unravel the mysteries of piezoelectricity and many-body effects in order to pursue a better engineering of the optical properties of InGaN QWs. ${ }^{7}$ Piezoelectricity occurs in a

${ }^{a)}$ Electronic mail: peng@cc.ee.ntu.edu.tw wurtzite-type QW due to a strain-induced polarization in the longitudinal (growth) direction, e.g., $c$ axis. ${ }^{8}$ The piezoelectric field for a low In composition epilayer on GaN typically measures a strength of $1 \mathrm{MV} / \mathrm{cm}$. In a strained InGaN QW, a novel piezoelectricity-induced quantum-confined Stark effect (PQCSE) has recently come to our attention. ${ }^{9}$ By taking advantage of such a novelty, one can further tailor the electronic $^{10,11}$ as well as the optical properties of nitridebased QW devices. ${ }^{12}$

Since the lasing and high brightness emission of $\mathrm{InGaN}$ QW take place at high levels of current injection, study of the many-body effects on the recombination process has become an important issue in the field. The many-body effects in a semiconductor emission include the band gap renormalization, plasma screening, and excitonic enhancement of the interband transition probability. ${ }^{13}$ However, we note that the excitonic phase in a highly excited QW becomes unstable ${ }^{14}$ as the two-dimensional electron-hole density exceeds $n_{c}$ $=1 / \pi a_{B}^{2}$, where $a_{B}$ is the exciton Bohr radius. When subject to an electric field, it is found that a field strength of 6 $\times 10^{5} \mathrm{~V} / \mathrm{cm}$ is sufficient to dissociate the free excitons in a nitride QW. ${ }^{15}$ Thus for a piezo-strained InGaN QW operated in a high carrier excitation regime, it has been shown to be sufficient to consider a dominant process of band-to-band transitions in analyzing the luminescence, ${ }^{16}$ optical gain, ${ }^{17}$ and lasing phenomena ${ }^{18}$ at room temperature.

In this letter, we report a series of electroluminescence (EL) experiments on strained $\mathrm{GaN} / \mathrm{InGaN} / \mathrm{AlGaN}$ single quantum well (SQW) light emitting diodes (LEDs) in which a pulsed current excitation technique has been used to study the QW emission in the high carrier injection regime. By analyzing the effects of PQCSE, band gap renormalization, 


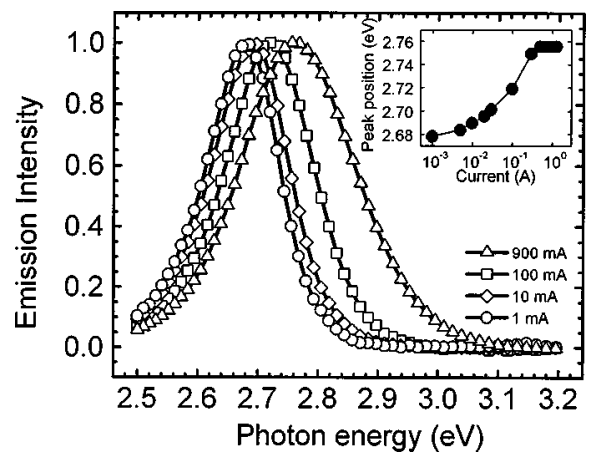

FIG. 1. Normalized EL spectra of a $3.0 \mathrm{~nm} \operatorname{In}_{0.2} \mathrm{Ga}_{0.8} \mathrm{~N}$ QW blue emitter at various injection currents.

band filling, and charge screening in a self-consistent scheme, we are able to quantify the phenomenon of carrierinduced spectral shifting in a strained InGaN QW for the first time. We therefore attribute the spectral blueshifting and enhancement of the QW emission intensity to the dominant charge screening and band-filling effects in a band-to-band recombination process.

Samples used in this study were grown by metalorganic chemical vapor deposition (MOCVD) on (0001) sapphire substrates; the active region of the SQW LED consisted of an undoped $3.0 \mathrm{~nm}$ strained $\operatorname{In}_{0.2} \mathrm{Ga}_{0.8} \mathrm{~N}$ epilayer sandwiched between a $n$-type $\mathrm{Si}: \mathrm{GaN}$ and a $p$-type $\mathrm{Mg}: \mathrm{Al}_{0.2} \mathrm{Ga}_{0.8} \mathrm{~N}$ layer of $4 \mu \mathrm{m}$ and $100 \mathrm{~nm}$ thickness, respectively. ${ }^{5}$ In order to investigate the LED emission spectrum under a high carrier injection condition, a pulsed current excitation technique was used in the measurement. The devices were biased with pulsed currents up to $1 \mathrm{~A}$ at a pulse width of $0.5 \mu \mathrm{s}$ and a low repetition rate of $10 \mathrm{~Hz}$. With a duty cycle as low as $10^{-5}$, one was able to eliminate the heating effects and avoid any artifacts in the spectrum analysis.

In Fig. 1 we illustrate the normalized EL spectra of the strained $3.0 \mathrm{~nm} \mathrm{In}_{0.2} \mathrm{Ga}_{0.8} \mathrm{~N}$ QW blue emitter at various injection currents. At room temperature, a large spectral blueshift of $80 \mathrm{meV}$ in the emission peak energy is resolved as the injection current increases from $1 \mathrm{~mA}$ to $1 \mathrm{~A}$. Spectral broadening also occurs with the increase of current injection. We note that the spectral blueshifting in a InGaN QW occurs opposite that of a InGaN double heterostructure. In the latter, one typically measures a spectral redshift under a high current injection condition. This has been ascribed to a band gap renormalization process resulting from the many-body effects. ${ }^{19}$ Since the effect of band gap renormalization increases with a reduced dimensionality, ${ }^{20}$ the spectral blueshifting in a InGaN QW emission represents an interesting fundamental phenomenon to be resolved. In addition, the so-called band gap narrowing ${ }^{5}$ upon which the emission peak energy of a InGaN QW is found considerably smaller than that of a stress-free InGaN epilayer is worth noting. ${ }^{21}$ Plausible causes of such a band gap narrowing have been accredited to the exciton and stress effects but no quantitative explanations have been made. ${ }^{5}$

Since the phenomena of band gap narrowing and spectral blueshifting are pertinent to the device operation of In$\mathrm{GaN}$ QW emitters, it is desirable to resolve these novelties from a fundamental point of view. One therefore has to apply a self-consistent analysis regarding the effects of PQCSE,

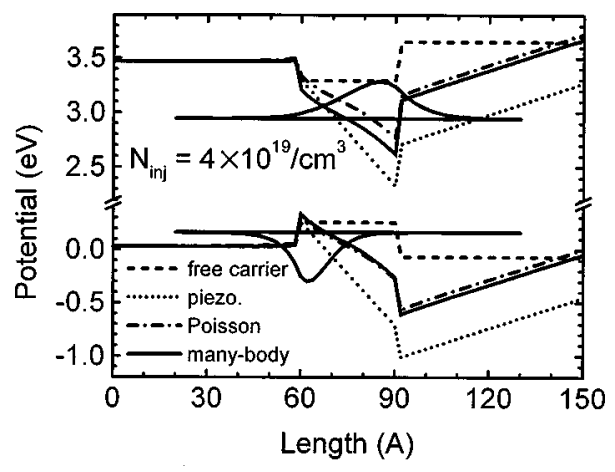

FIG. 2. Evolution of the GaN/InGaN/AlGaN QW potential profiles as each of the perturbations is sequentially turned on. The injection carrier concentration $N_{\text {inj }}$ is assumed to be $4 \times 10^{19} \mathrm{~cm}^{-3}$.

band gap renormalization, band filling, and charge screening on the optical properties of InGaN QWs. In doing so, the equation of motion for the conduction band Schrödinger equation and valence band RSP Hamiltonian are solved iteratively by coupling with a Poisson equation whose spatially varying piezoelectric field is concurrently screened by a charge distribution in the $\mathrm{QW}$ region. The two-dimensional band gap renormalization of a InGaN QW is solved by following the formalism described in Ref. 22. The material parameters of InGaN and $\mathrm{AlGaN}$ are taken as a linear interpolation from those of the binary $\mathrm{GaN},{ }^{23} \mathrm{AlN},{ }^{24}$ and $\mathrm{InN},{ }^{25}$ except that the deformation potentials of AlN and $\mathrm{InN}$ are assumed to be the same as those of GaN.

In Fig. 2 we depict the corrected potential profiles of a 3 nm strained $\mathrm{GaN} / \mathrm{In}_{0.2} \mathrm{Ga}_{0.8} \mathrm{~N} / \mathrm{Al}_{0.2} \mathrm{Ga}_{0.8} \mathrm{~N} \mathrm{QW}$ and compare it with a free-carrier flatband model calculation as each of the fundamental interactions is sequentially turned on. The comparison is made with an injected carrier density $N_{\text {inj }}$ of 4 $\times 10^{19} \mathrm{~cm}^{-3}$ in the InGaN well region. Data shown in Fig. 2 reveal how critically the QW potential profile evolves with the perturbations and indicates the importance of performing a self-consistent analysis. Due to the charge screening (Poisson) and band gap renormalization effects, we note the effective strength of the piezoelectric field is reduced from 3.21 to $1.96 \mathrm{MV} / \mathrm{cm}$. We also illustrate in Fig. 2 the $k_{\|}=0$ subband envelope functions of $n=1$ conduction $\left(C_{1}\right)$ and heavy hole $\left(\mathrm{HH}_{1}\right)$ states. A reduction in the oscillator strength has taken place as the spatial overlapping between the electron and hole is decreased by the piezoelectric field.

In Fig. 3 we calculate the optical gain of the strained 3.0 $\mathrm{nm}$ InGaN QW following the scenario outlined in Fig. 2.

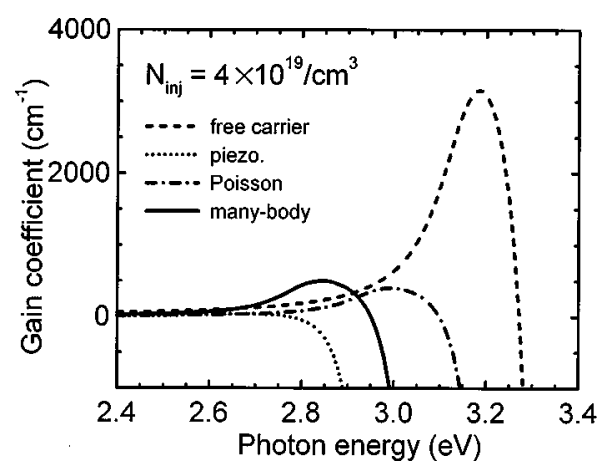

FIG. 3. Calculated optical gain of the $3.0 \mathrm{~nm} \mathrm{GaN} / \mathrm{InGaN} / \mathrm{AlGaN}$ QW. 


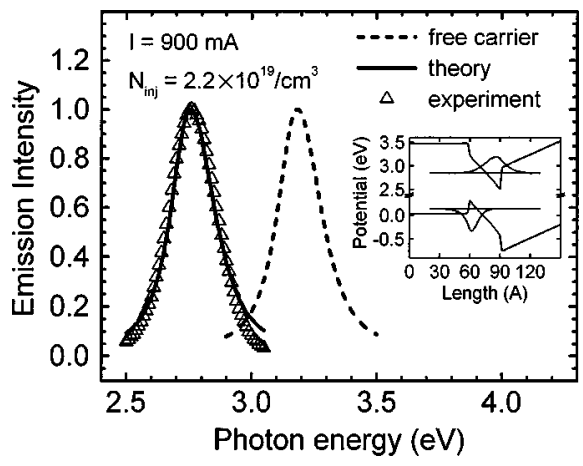

FIG. 4. Comparison between the calculated optical emission spectra and the experimental data. The experimental data are from Fig. 1 at a pulsed injection current of $900 \mathrm{~mA}$.

Since the selection rule of forbidden interband transitions $(\Delta n \neq 0)$ relaxes in a QW under a strong electric field,${ }^{26}$ it is sufficient to take a summation over two conduction and seven valence subbands in evaluating the optical gain of a narrow $\mathrm{In}_{0.2} \mathrm{Ga}_{0.8} \mathrm{~N} / \mathrm{Al}_{0.2} \mathrm{Ga}_{0.8} \mathrm{~N} \mathrm{QW}$ studied in this case. ${ }^{27} \mathrm{By}$ doing so we can quantitatively differentiate the contributions of PQCSE, charge screening, band filling, and band gap renormalization to the optical gain of a strained $\mathrm{InGaN} \mathrm{QW}$. Data shown in Fig. 3 clearly reveal the fact that a free carrier model calculation indeed exaggerates the optical gain of a InGaN QW while a piezoelectric calculation without taking into account the charge screening effect can severely underestimate the result. Under the influence of charge screening and band gap renormalization, we notice that a more profound quantum confinement has been established with respect to the unscreened case.

Finally Fig. 4 shows the calculated spontaneous emission spectra of the $3.0 \mathrm{~nm} \mathrm{InGaN} \mathrm{blue} \mathrm{emitter} \mathrm{in} \mathrm{comparison}$ with the experimental data obtained under a pulsed current excitation at $900 \mathrm{~mA}$. In the self-consistent analysis, only the injected carrier density $\left(N_{\mathrm{inj}}=2.2 \times 10^{19} \mathrm{~cm}^{-3}\right)$ and the linewidth broadening factor ${ }^{28}\left(1 / \tau=120 \mathrm{ps}^{-1}\right)$ are chosen as the fitting parameters. We have found good agreement between the calculation and the experiment over a wide spectral range of $0.5 \mathrm{eV}$. We thereby can rationalize the so-called band gap narrowing 5 as being due to the omission of PQCSE, charge screening, and band gap renormalization in the commonly used free-carrier model analysis. ${ }^{29}$ Moreover, we note there exists a minor discrepancy in the low energy portion $(\sim 2.5$ $\mathrm{eV}$ ) of the calculated emission spectrum with respect to the experimental data. The latter might result from neglecting the band tail states in the analysis. ${ }^{30}$

In summary, we report a high current injection analysis of the EL spectra of strained $\mathrm{GaN} / \mathrm{In}_{0.2} \mathrm{Ga}_{0.8} \mathrm{~N} / \mathrm{Al}_{0.2} \mathrm{Ga}_{0.8} \mathrm{~N}$ SQW LEDs. A spectral blueshift of $80 \mathrm{meV}$ is observed as the injection current increases from $1 \mathrm{~mA}$ to $1 \mathrm{~A}$. The emission spectrum of a strained InGaN QW is determined by a competition between a spectral redshifting mechanism of PQCSE and band gap renormalization, and a blueshifting mechanism of band-filling and Poisson (charge screening) effects. In the high carrier injection regime, a self-consistent model analysis has been proposed and shown excellent agreement with the experiment. The increase of InGaN QW emission peak energy and intensity with injected carriers suggests a dominant band-to-band recombination process at room temperature.

The authors acknowledge discussion with Professor H. Ehrenreich at Harvard University. This research was partially supported by the National Science Council, Grant No. 88-2212-M-002-032 and by the R. O. C. Department of Economy under Contract No. 88S7-T1.

${ }^{1}$ S. Nakamura, IEEE J. Sel. Top. Quantum Electron. 3, 712 (1997).

${ }^{2}$ S. Nakamura, M. Senoh, N. Iwasa, and S. I. Nagahama, Appl. Phys. Lett. 67, 1868 (1995).

${ }^{3}$ S. Nakamura, M. Senoh, S. I. Nagahama, N. Iwasa, T. Yamada, T. Matsushita, H. Kiyoku, Y. Sugimoto, T. Kozaki, H. Umemoto, M. Sano, and K. Chocho, Jpn. J. Appl. Phys., Part 2 36, L1568 (1997).

${ }^{4}$ S. Nakamura, M. Senoh, S. I. Nagahama, N. Iwasa, T. Yamada, T. Matsushita, H. Kiyoku, Y. Sugimoto, T. Kozaki, H. Umemoto, M. Sano, and K. Chocho, Appl. Phys. Lett. 72, 2014 (1998).

${ }^{5}$ S. Nakamura, IEEE J. Sel. Top. Quantum Electron. 3, 435 (1997).

${ }^{6}$ Y. M. Sirenko, J. B. Jeon, B. C. Lee, K. W. Kim, M. A. Littlejohn, M. A. Stroscio, and G. J. Iafrate, Phys. Rev. B 55, 4360 (1997).

${ }^{7}$ W. W. Chow, S. W. Loch, and M. Sargent III, Semiconductor Laser Physics (Springer, Berlin, 1993).

${ }^{8}$ A. Bykhovski, B. Gelmont, and M. Shur, J. Appl. Phys. 74, 6734 (1993).

${ }^{9}$ T. Takeuchi, S. Sota, M. Katsuragawa, M. Komori, H. Takeuchi, H. Amano, and I. Akasaki, Jpn. J. Appl. Phys., Part 2 36, L382 (1997).

${ }^{10}$ E. T. Yu, G. J. Sullivan, P. M. Asbeck, C. D. Wang, D. Qiao, and S. S. Lau, Appl. Phys. Lett. 71, 2794 (1997).

${ }^{11}$ R. Gaska, J. W. Yang, A. Osinsky, A. D. Bykhovski, and M. S. Shur, Appl. Phys. Lett. 71, 3673 (1997).

${ }^{12}$ J. S. Im, H. Kollmer, J. Off, A. Sohmer, F. Scholz, and A. Hangleiter, Phys. Rev. B 57, R9435 (1998).

${ }^{13}$ W. W. Chow, A. F. Wright, A. Girndt, F. Jahnke, and S. W. Koch, Appl. Phys. Lett. 71, 2608 (1997).

${ }^{14}$ S. L. Chuang, IEEE J. Quantum Electron. 32, 1791 (1996).

${ }^{15}$ S. Chichibu, T. Azuhata, T. Sota, and S. Nakamura, Appl. Phys. Lett. 69, 4188 (1996)

${ }^{16}$ C. K. Sun, S. Keller, T. L. Chiu, G. Wang, M. S. Minsky, J. E. Bower, and S. P. DenBaars, IEEE J. Sel. Top. Quantum Electron. 3, 731 (1997).

${ }^{17}$ G. Frankowsky, F. Steuber, V. Harle, F. Scholz, and A. Hangleiter, Appl. Phys. Lett. 68, 3746 (1996)

${ }^{18}$ K. Domen, A. Kuramata, and T. Tanahashi, Appl. Phys. Lett. 72, 1359 (1998)

${ }^{19}$ G. Y. Zhao, G. Yu, T. Egawa, J. Watanabe, T. Jimbo, and M. Umeno, Appl. Phys. Lett. 71, 2424 (1997).

${ }^{20}$ G. Trankle, H. Leier, A. Forchel, H. Haug, C. Ell, and G. Weimann, Phys. Rev. Lett. 58, 419 (1987).

${ }^{21}$ S. Nakamura, Solid State Commun. 102, 237 (1997).

${ }^{22}$ S.-H. Park and S.-L. Chuang, Appl. Phys. Lett. 72, 287 (1998).

${ }^{23}$ Properties of Group III Nitrides, edited by J. H. Edgar (INSPEC/IEE, London, 1994).

${ }^{24}$ S. Nakamura and G. Fasol, The Blue Laser Diode (Springer, Berlin, 1997).

${ }^{25}$ Y. C. Yeo, T. C. Chong, and M. F. Li, J. Appl. Phys. 83, 1429 (1998).

${ }^{26}$ D. A. B. Miller, J. S. Weiner, and D. S. Chemla, IEEE J. Quantum Electron. 22, 1816 (1986)

${ }^{27}$ L.-H. Peng and C.-W. Chuang (unpublished).

${ }^{28}$ K. P. O'Donnel, T. Breitkopf, H. Kalt, W. Van der Stricht, I. Moerman, P. Demeester, and P. G. Middleton, Appl. Phys. Lett. 70, 1843 (1997).

${ }^{29}$ J. Wang, J. B. Jeon, Yu. M. Sirenko, and K. W. Kim, IEEE Photonics Technol. Lett. 9, 728 (1997).

${ }^{30}$ P. G. Eliseev, P. Perlin, J. Lee, and M. Osinski, Appl. Phys. Lett. 71, 569 (1997). 